\title{
The Effect of Hydrogen Cyanamide on Dormancy Breaking In Grapevine Buds: Reactive Oxygen Species Accumulation and Related Genes Expression
}

\author{
Boonyawat Sudawan', Chih-Sheng Chang ${ }^{2}$, Hsiu-fung Chao ${ }^{3}$, Maurice S. B. Ku ${ }^{4}$, Yung-fu Yen ${ }^{5}$ \\ ${ }^{1} \mathrm{Ph}$.D. Program of Agricultural Science, National Chiayi University, \\ 300, University Road, Chiayi City, Taiwan \\ bsudawan27@gmail.com \\ ${ }^{2}$ Department of Farmers' Services, Council of Agriculture, Taipei. Taiwan \\ 37, Nanhai Road, Taipei City, Taiwan \\ changcs@mail.coa.gov.tw \\ ${ }^{3}$ Tainan District Agricultural Research and Extension Station \\ 70 Muchang, Hsinhua District, Tainan City, Taiwan \\ hfchao@mail.tndais.gov.tw \\ ${ }^{4}$ Dept. of Bioagricultural Science, National Chiayi University, \\ 300, University Road, Chiayi City, Taiwan \\ mku@mail.ncyu.edu.tw \\ ${ }^{5}$ Dept. of Bioagricultural Science, National Chiayi University, \\ 300, University Road, Chiayi City, Taiwan \\ yfyen@mail.ncyu.edu.tw
}

\begin{abstract}
Hydrogen cyanamide (HC) and pruning have frequently been used to break dormancy in grapevine floral buds. This study aimed to address the effects of these treatments on accumulation of ROS and alteration in expression of ROS-related genes in the dormancy breaking buds of grapevine in the summer in sub/tropical. Four treatments were compared, namely: Pruning (P), hydrogen cyanamide (HC), Pruning and hydrogen cyanamide (PHC) and Control (water) and 8 days after treatment the bud break rates were 33\%, $53 \%, 95 \%$, and $0 \%$, respectively. Clearly, $\mathrm{HC}$ is effective in stimulating grapevine bud break and pruning further enhanced its potency. In situ detection of various Reactive oxygen species (ROS) and Nitric oxide (NO) in longitudinal bud sections after $12 \mathrm{~h}$ of treatments showed that high levels of ROS and NO accumulate in the buds treated with PHC, compared with HC or P only. The amounts of ROS and $\mathrm{NO}$ accumulated were closely correlated with the rates of bud break among the treatments. Microarray analysis was conducted with the dormancy breaking buds after $24 \mathrm{~h}$ of treatments. Gene ontology (GO) analysis indicated that alteration in expression of ROS related genes is the major factor responsible for bud break. PHC treatment gave rise to dynamic changes in highly up-/down-regulation of antioxidant activity at $24 \mathrm{~h}$ post-treatment. Twelve genes were identified as key genes involved in dormancy bud break in the early response. The time course of expression of these genes (examined by qRT-PCR) showed different expression during the $48 \mathrm{~h}$ treatment. It is concluded that accumulation of ROS at the early stage is important for dormant bud break, and that the microarray analy sis of differentially expressed genes among the treatments allowed the construction of the model pathway related to ROS metabolisms during dormant bud breaking.
\end{abstract}

Keywords: Hydrogen cyanamide, ROS, dormancy breaking buds, grapevines, Gene Ontology

\section{Introduction}

Grapevine is a perennial crop in temperate climates and perceives cool temperatures as a signal to onset bud endodormancy while entering winter; subsequently it requires chilling temperature for several weeks (or months) to end of endodormancy of these buds [1]. Grapevine grown in subtropical regions, often have an uneven floral bud break in early spring due to warm winter that providing inadequate chilling. Fortunately, $\mathrm{HC}$ have been found to be one of the most useful compounds for breaking dormancy in floral buds in grapevine [2-4], kiwifruit [5] and apple [6, 7]. In subtropical regions the use of $\mathrm{HC}$ treatment on intact dormant buds in spring is an important grapevine orchard management tool to regulate for 
even floral bud break. This treatment is also very useful in Taiwan for the second grapevine harvest in December, where paradormant buds on the prune canes can be treated with HC to ensure effective bud break even in hot August.

To gain a better understanding of the underlying mechanism of bud break by pruning and/or HC treatment, this study aimed to observe the burst out of endogenous ROS and NO levels in the dormancy breaking buds by cytochemical staining and to identify the sets of candidate genes being significantly altered by microarray analysis. Go categories that related to ROS-generating, ROS- scavenging, such as peroxidase activity (GO:0004601), antioxidant activity (GO: 0016209) and NO detoxification were identified as key genes involved in breaking dormancy of grapevine buds in early response and their expression patterns during treatment were subsequently analysed by qRT-PCR analysis.

\section{Materials and Methods \\ Plant material}

The grapevine buds used in this experiments in August were collected from the mature canes of 5-year-old plants (V. vinifera. x V. labruscana Bailey cv. Kyoho) grown in the research vineyard of the Taichung District Agricultural Research

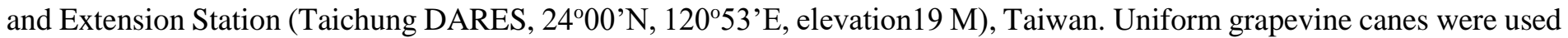
for four treatments with water only (as control; CK), pruning only (P), 1\% (w/v) of HC (Dormex, SKW, Trostberg, Germany) and a combination of pruning and $\mathrm{HC}$ (PHC).

Observation of bud developments after treatments by light microscopy

Grapevine buds were collected before and after treatments and fixed in FAA solution for $2 \mathrm{~h}$, stained with filtered $0.1 \%$ Harris Hematoxylin solution, Images of sections were captured.

\section{In situ $\mathrm{H2O}$, $\mathrm{O2}^{*-}$ and NO detection by fluorescence microscopy}

For in situ staining assay of $\mathrm{H}_{2} \mathrm{O}_{2}, \mathrm{O}_{2}{ }^{-}$and $\mathrm{NO}$, dormant grapevine buds treated with $\mathrm{P}, \mathrm{HC}, \mathrm{PHC}$ and $\mathrm{CK}$ for $12 \mathrm{~h}$

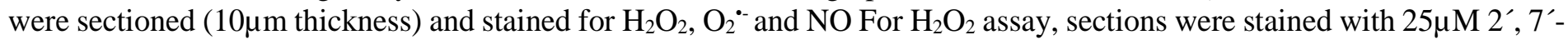
dichlorofluorescin diacetate (DCF-DA, Calbiochem, San Diego, CA, USA) [8]. For $\mathrm{O}_{2}{ }^{\circ-}$ assay, sections were stained with $10 \mu \mathrm{M}$ dihydroethidium (DHE, Calbiochem, San Diego, CA, USA) [9]. For NO on the sections were stained with 10 $\mu \mathrm{M} 4$, 5-diaminonaphthalene (DAF-2 DA, Calbiochem, San Diego, CA, USA) prepared in $10 \mathrm{mM}$ Tris- HCl (pH 7.4). For negative controls, sections were incubated sequentially with $1 \mathrm{mM}$ sodium pyruvate $\left(\mathrm{H}_{2} \mathrm{O}_{2}\right.$ scavenger $), 1 \mathrm{mM}$ tetramethylpiperdinooxy $\left(\mathrm{O}_{2}{ }^{-}\right.$scavenger), and $1 \mathrm{mM}$ carboxy-PTIO(NO inhibitor).

\section{RNA extraction and microarray analysis}

Microarray experiment procedures were carried out following the manufacturer's protocols. Briefly, $1 \mu \mathrm{g}$ of total RNA was amplified by a Agilent Qiuck Amp Labeling Kit (Agilent Technologies,USA) and labeled with Cy3-CTP or Cy5-CTP (Agilent Technologies, USA). Correspondingly fragmented labeled cRNA is then pooled and hybridized to Agilent Whole Grape Genome 4x44k oligo microarray (Agilent Technologies, USA). Scanned images are analyzed by Feature extraction software 10.5(Agilent Technologies, USA), finally, the GenSpring software (Agilent Technologies, USA) is used to identify of significant differentially expressed genes (DEGs).

\section{Differentially expressed gene annotation and functional categorization}

Gene Ontology (GO) analysis for gene information annotation and functional category distribution frequency was performed using the ErmineJ (version 3.0.2) software [10].

\section{Quantification of gene expression by quantitative RT- PCR (qRT-PCR)}

Total RNA was extracted at $0,6,12,24,48 \mathrm{~h}$ post-treatment and 12 grapevine genes were selected for quantification of gene expression by quantitative RT-PCR.

\section{Results and Discussion \\ Dormant bud break rates}

In this study we showed that intact pruned $(\mathrm{P})$ in combination with $\mathrm{HC}$ (PHC) treatment effectively alleviate the dormancy of grapevine buds and result in bud break and floral development in hot summer (Figure 1A). PHC induces bud break more rapidly within 8 days and efficiently $(95 \%)$ than $\mathrm{P}$ or HC treatment alone, indicating a synergetic interaction between $\mathrm{P}$ and HC treatments (Figure 1B). A similar result has been reported previously in grapevine [11]. Compared to dormant buds, PHC treated buds showed a bud break mediated by paradormancy, in which growth is regulated by plant growth regulators originating outside the bud affecting apical dominance [12]. In contrast to paradormancy that occurs in the summer, endodormancy that occurs during winter is a major problem for grape production in warm winter region [13, 14]. 
(A)

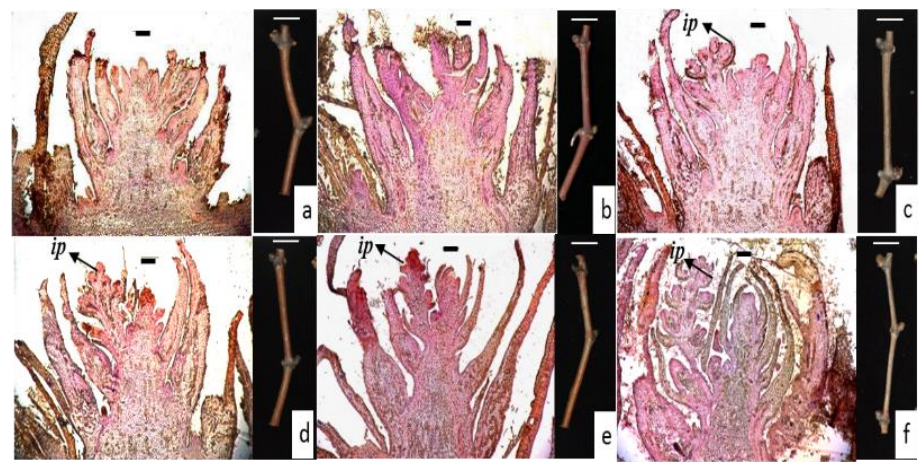

(B)

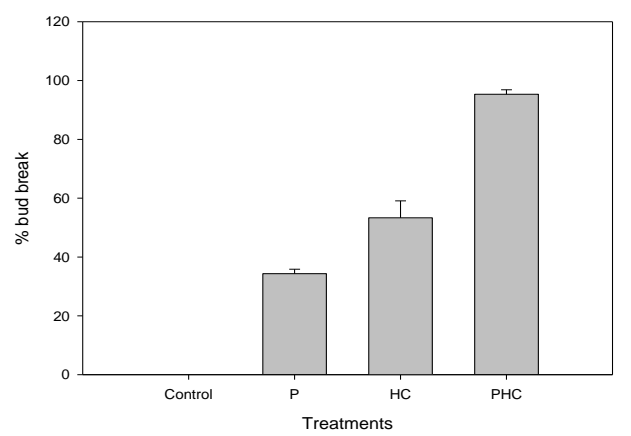

Fig. 1: Morphological observation and anatomical examination of grapevine bud break. (A) Longitudinal sections of grapevine floral development bud during breaking of dormancy after PHC treatment. (a) control (0 h), (b) $6 \mathrm{~h}$, (c) $12 \mathrm{~h}$, (d) $24 \mathrm{~h}$, (e) $48 \mathrm{~h}$ and (f) $96 \mathrm{~h}$. ip: inflorescence primordium. Bar: $200 \mu \mathrm{m}$. (B) Percentages of bud break after $192 \mathrm{~h}$ of treatment with P, HC, PHC, or water as a control ( $\mathrm{n}=10$, Bar: standard deviation).

\section{In situ $\mathrm{H}_{2} \mathrm{O}_{2}, \mathrm{O}_{2}{ }^{*-}$ and $\mathrm{NO}$ detection by fluorescence microscopy}

In this study, we detected large amounts of $\mathrm{H}_{2} \mathrm{O}_{2}, \mathrm{O}_{2}{ }^{-}$and $\mathrm{NO}$ levels accumulated rapidly in bud tissues by in situ localization upon the treatments, especially in PHC treated buds (Figure 2).

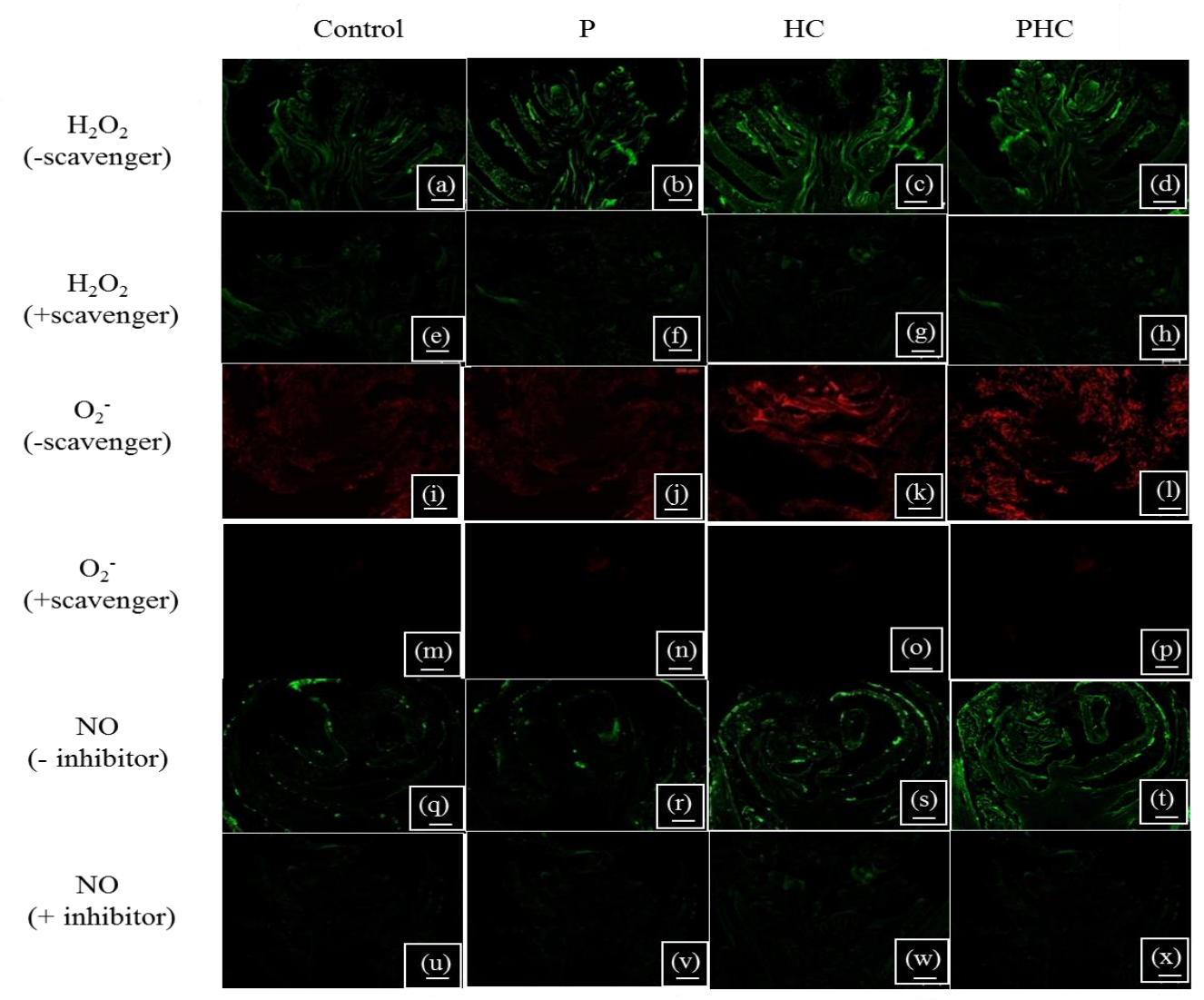

Fig. 2: Detection of $\mathrm{H} 2 \mathrm{O} 2, \mathrm{O} 2 \bullet-$ and $\mathrm{NO}$ at early stage of grapevine bud break.

Visualization of $\mathrm{H} 2 \mathrm{O} 2$ by fluorescence microscopy using DCF-DA assay in grapevine bud sections after $12 \mathrm{~h}$ of treatment with $\mathrm{H} 2 \mathrm{O}$ or control (a), P (b), $\mathrm{HC}$ (c) and PHC (d). For negative control, grapevine bud sections were incubated with $1 \mathrm{mM}$ sodium pyruvate, an $\mathrm{H} 2 \mathrm{O} 2$ scavenger: control (e), P (f), HC (g) and PHC (h). Visualization of O2•- by reaction 
with $10 \mu \mathrm{M}$ dihydroethidium (DHE) in grapevine bud sections: H2O or control (i), P (j), HC (k) and PHC (l). For negative control, grapevine bud sections were incubated in $1 \mathrm{mM}$ tetramethylpiperdinooxy, an O2•- scavenger: control (m), P (n), HC (o) and PHC (p). Visualization of nitric oxide (NO) by DAF-2DA assay in grapevine bud sections: control (q), P (r), HC (s) and PHC (t). For negative control, grapevine bud sections were incubated in $10 \mu \mathrm{M}$ carboxy-PTIO, an NO inhibitor: control (u), P (v), HC (w) and PHC (x). Bar: $200 \mu \mathrm{m}$.

\section{Classification of the expression of up/down regulated genes that related to ROS in three treatment at 24 post- treatment}

The expressed genes that were significantly regulated by the three treatments were identified by GenSpring. It is noteworthy that a total of 1148, 2251 and 4058 genes showed up-regulated expression and a total of 206, 1169 and 3115 genes showed down-regulated expression by P, HC and PHC treatments, respectively. Clearly, alteration in expression of many genes is involved in dormant bud break in grapevine and many more genes are up-regulated than down-regulated by these treatments.

GO classification of ROS related genes that up-/down regulated in molecular function at $24 \mathrm{~h}$ post-treatment, these genes showing altered expression in ROS- and NO-related. We identified clear overlaps of the molecular functions among the up-/down-regulated genes in responses to P, HC and PHC. GO term were showed its individual expression levels by significantly at least two fold in up-/down-regulated changes in each treatment.

However, PHC treatment was mostly induced up-/down-regulated genes following HC and P treatment. The specific of $\mathrm{P}$ and $\mathrm{HC}$ response are suggests that there are common regulation of genes response to PHC. ROS-generating genes; respiratory burst oxidase homolog protein E; VvRBOHE, respiratory burst oxidase homolog protein A; VvRBOHA, VvPOD72, VvPOD12 and VvDOX were identified. Two-VvRBOH were appeared in binding (GO:0005488), which both VvRBOHE and VvRBOHA were showed down-regulated of their expression in PHC, and one-VvRBOH was downregulated in HC. VvPOD and VvDOX were appeared in peroxidase activity (GO:0004601), which the VvPOD genes; that mention are ROS-generating genes have been expressed in up-/down-regulated genes in P (10/1), HC (10/14) and PHC (15/10). One VvDOX gene was up-regulated in P and HC, which was not found in PHC. For ROS-scavenging genes were including in antioxidant activity (GO:0016209), which totally four genes family have been expressed in up-/down regulated along three treatments; such as one glutathione peroxidase; VvGPX gene was up-regulated in PHC and the another one VvGPX gene was down-regulated in HC and PHC, while two catalase isozyme -like; VvCAT genes were down-regulated in both HC and PHC. In addition, cytosolic ascorbate peroxidase; VvAPX was found in down-regulated in HC and PHC, superoxide dismutase [Fe], chloroplastic; VvFSD3 was down-regulated in HC and PHC and VvFSD was only up-regulated in PHC. VvAOX was up-regulated in HC and PHC. For NO relative gene, hemoglobin-2; VvHB2 was including in binding (GO:0005488) by up-regulated expression in HC and PHC. ROS-transcription factor, four gene families; heat stress transcription factor; VvHSF, ethylene responsive transcription factor; VvERF and WRKY transcription factor; VvWRKY were found in transcription factor (GO:0003700). The number of VvHSF genes were up-/down-regulated by P (0/1), HC (2/2), and PHC (3/6), while the VvERF genes were up-/down-regulated expressed in P (4/3), HC (8/6), and PHC (8/9). VvWRKY genes were up-/down-regulated in P (1/0), HC (9/4), PHC (7/7), respectively.

\section{Detection of ROS-generating, ROS- scavenging and NO-scavenging genes}

Consistent with this observation, the expression of genes coding for five key ROS-generating genes, were both induced (VvPOD72, VvDOX) and suppressed (VvRBOHE, VvRBOHA, VvPOD12) at the early stage of PHC treatment. In addition, ROS-scavenging genes, such as VvCAT and VvFSD3 were suppressed at the early stage of PHC treatment (Fig 3), thus allowing the rapid accumulation of related ROS.

As reported in grape buds after 12-24 h of hydrogen cyanide treatment was reduced CAT activity and expression, but was induced up-regulated of oxidative stress related genes, such as $\operatorname{Tr} x h, G S T, A P X, G R$, and hypoxia related genes, such as SuSy $[4,15,16]$. A common mode of ROS regulation gene networks and its modulation in grapevine dormant buds break are identified their expression profiles during PHC treatment by qRT-PCR. Totally 12 genes were identified by qRT-PCR; six genes were related to ROS-scavenging genes, such as $V v F S D 3$ and $V v A O X$ are turned on and expressed at high level at $6 \mathrm{~h}$, while $V v G P X$ and $V v A P X$ were turned on and expressed at high level as early as $12 \mathrm{~h}$ of treatment and decline at 24-48 h, except $V v C A T$ and $V v F S D 3$ which were down-regulated.

For RNS detoxification we have found the expression of $V v H B 2$ is highest expression at $6 \mathrm{~h}$ post-treatment by PHC, which $A H b 1$ is a member of the class I family of nonsymbiotic hemoglobins [17], and its possessing activity against NO [18] and $\mathrm{AHb} 1$ expression is induced in roots and rosette leaves by low $\mathrm{O} 2$ levels [19]. Therefore, we recommend the $V v H B 2$ 
was early highest expression at $6 \mathrm{~h}$ post-treatment which can be inhibited the NO accumulation that further be able damaging the cell.
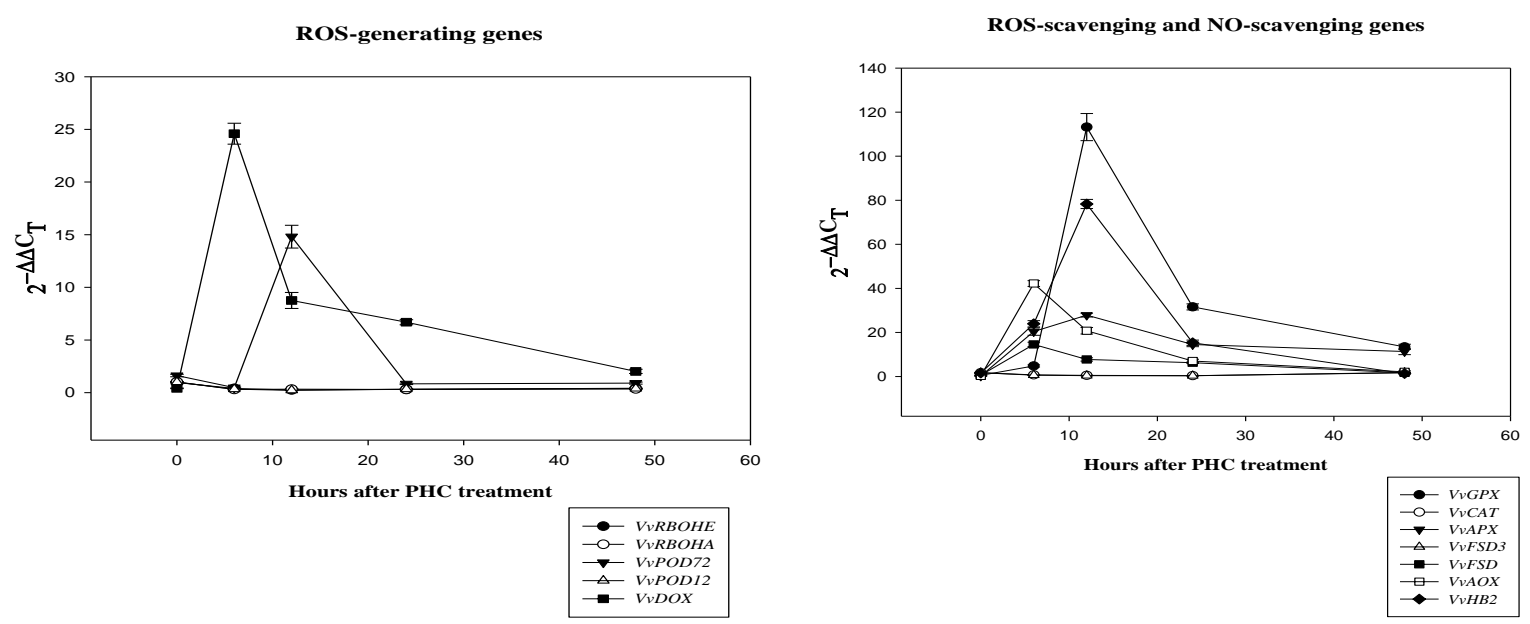

Fig. 3: The gene expression among the 12 up-/down-regulated ROS-relative genes induced by PHC. During of $48 \mathrm{~h}$ treatment, as analyzed by qRT-PCR, ROS-generating genes; (A) VvRBOHE, VvRBOHA, VvPOD72, VvPOD12 and (B) VvDOX. ROS-scavenging genes; VvGPX, VvCAT, VvAPX, VvFSD3, VvFSD, VvAOX and NO-scavenging genes; VvHB2.

\section{Conclusions}

In concluded, this study is contributed our understanding to underlying in ROS metabolism pathway. According to hydrogen cyanamide is applied to grapevine dormant buds, its need ROS-mediated to breaking dormant buds. The upstream events, likely ROS generating and ROS accumulation in the cell were induced the oxidative stress and trigger programmed cell death response in the early stage (e.g. 6-12 h) of hydrogen cyanamide treatment in grapevine buds. In addition, the interaction of ROS and NO can be correlated in activating dormancy breaking in grapevine buds.

\section{Acknowledgements}

The authors acknowledge the supports of the Ph.D. Program of Agricultural Science National Chiayi University and also thank Ermery M. Ku for critical reading and revised the manuscript.

\section{References}

[1] K. Mathiason, D. He, J. Grimplet, J. Venkateswari, D. W. Galbraith, E. Or, and A. Fennell, "Transcript profiling in Vitis riparia during chilling requirement fulfillment reveals coordination of gene expression patterns with optimized bud break," Funct \& Integr Genomics, vol. 9, pp. 81-96, 2009.

[2] Y. Shulman, G. Nir, L. Fanberstein, and S. Lavee, "The effect of cyanamide on the release from dormancy of grapevine buds," Sci Hortic, vol. 19, no. 1, pp. 97-104, 1983.

[3] E. Or, I. Vilozny, Y. Eyal, and A. Ogrodovitch, "The transduction of the signal for grape bud dormancy breaking induced by hydrogen cyanamide may involve the SNF-like protein kinase GDBRPK," Plant Mol Biol, vol. 43, pp. 483-494, 2000.

[4] R. Ophir, X. Pang, T. Halaly, J. Venkateswari, S. Lavee, D. Galbraith, and E. Or, "Gene-expression profiling of grape bud response to two alternative dormancy-release stimuli expose possible links between impaired mitochondrial activity, hypoxia, ethylene-ABA interplay and cell enlargement," Plant Mol Biol, vol. 71, pp. 403-423, 2009.

[5] G. Linsley-Noakes, ÈImproving flowering of kiwifruit in climatically marginal areas using hydrogen cyanamide," Sci Hortic, vol. 38, pp. 247-259, 1989.

[6] J. Petri and H. Stuker, "Effect of mineral oil and hydrogen cyanamide concentrations on apple dormancy," Acta Hort, pp. 161-168, 1994.

[7] J. Jackson and M. Bepete, "The effect of hydrogen cyanamide (Dormex) on flowering and cropping of different apple cultivars under tropical conditions of sub-optimal winter chilling," Sci Hortic, vol. 60, pp. 293-304, 1995. 
[8] M. M. Tarpey, D. A. Wink, and M. B. Grisham, "Methods for detection of reactive metabolites of oxygen and nitrogen: in vitro and in vivo considerations," American Journal of Physiology-Regulatory, Integrative and Comparative Physiology, vol. 286, pp. R431-R444, 2004.

[9] Y. Yamamoto, Y. Kobayashi, S. R. Devi, S. Rikiishi, and H. Matsumoto, "Aluminum toxicity is associated with mitochondrial dysfunction and the production of reactive oxygen species in plant cells," Plant physiol, vol. 128, pp. 63$72,2002$.

[10] H. K. Lee, W. Braynen, K. Keshav, and P. Pavlidis, "ErmineJ: tool for functional analysis of gene expression data sets," BMC bioinformatics, vol. 6, pp. 269, 2005.

[11] R. Cirami and D. Furkaliev, "Effect of time of pruning and hydrogen cyanamide on growth and development of glasshouse-grown cardinal grapes," Anim Prod Sci, vol. 31, pp. 273-278, 1991.

[12] G. A. Lang, "Dormancy: a new universal terminology," HortScience, vol. 22, pp. 817-820, 1987.

[13] S. Lavee and P. May, "Dormancy of grapevine buds-facts and speculation," Aust J Grape Wine Res, vol. 3, pp. 31-46, 1997.

[14] N. Dokoozlian, "Chilling Temperature and Duration Interact on the Budbreak of Perlette'Grapevine Cuttings," HortScience, vol. 34, pp. 1-3, 1999.

[15] T. Keilin, X. Pang, J. Venkateswari, T. Halaly, O. Crane, A. Keren, A. Ogrodovitch, R. Ophir, H. Volpin, and D. Galbraith, "Digital expression profiling of a grape-bud EST collection leads to new insight into molecular events during grape-bud dormancy release," Plant Sci, vol. 173, pp. 446-457, 2007.

[16] T. Halaly, X. Pang, T. Batikoff, O. Crane, A. Keren, J. Venkateswari, A. Ogrodovitch, A. Sadka, S. Lavee, and E. Or, "Similar mechanisms might be triggered by alternative external stimuli that induce dormancy release in grape buds," Planta, vol. 228, pp. 79-88, 2008.

[17] B. Trevaskis, R. A. Watts, C. R. Andersson, D. J. Llewellyn, M. S. Hargrove, J. S. Olson, E. S. Dennis, and W. J. Peacock, "Two hemoglobin genes in Arabidopsis thaliana: the evolutionary origins of leghemoglobins," in Proc Natl Acad Sci, USA, 1997, vol. 94, pp. 12230-12234.

[18] M. Perazzolli, P. Dominici, M. C. Romero-Puertas, E. Zago, J. Zeier, M. Sonoda, C. Lamb, and M. Delledonne, "Arabidopsis nonsymbiotic hemoglobin AHb1 modulates nitric oxide bioactivity," Plant Cell, vol. 16, pp. 2785-2794, 2004.

[19] J. B. Wittenberg, M. Bolognesi, B. A. Wittenberg, and M. Guertin, "Truncated hemoglobins: a new family of hemoglobins widely distributed in bacteria, unicellular eukaryotes, and plants," J Biol Chem, vol. 277, pp. 871-874, 2002. 